\section{Análise eletromiográfica dos músculos da coxa no exercício agachamento afundo até a exaustão}

\section{Thigh muscles electromyographic activity during lunge exercise to fatigue}

\author{
Gisele Rodrigues Leite Garcia ${ }^{1}$ \\ Nuno Manuel Frade de Sousa ${ }^{2}$ \\ Vivian Maria Arakelian ${ }^{2}$ \\ Jéssica Fernanda Garcia \\ Runer Augusto Marson \\ Sérgio Eduardo de Andrade Perez \\ Vilmar Baldissera ${ }^{1,2}$
}

Resumo - O agachamento afundo possui um posicionamento dos membros inferiores diferencial em relação ao agachamento padrão, necessitando de maiores esclarecimentos acerca das participações dos músculos envolvidos. O objetivo foi analisar a atividade eletromiográfica dos músculos vastus lateralis $(\mathrm{VL})$, vastus medialis $(\mathrm{VM})$, bíceps femoris $(\mathrm{BF})$ e semitendinosus (ST) durante a execução do agachamento afundo até à exaustão com o membro inferior posicionado frontalmente e posteriormente. Participaram do estudo nove mulheres ativas com média (DP) de idade de $22(3,4)$ anos e massa corporal 60,3 $(4,1) \mathrm{kg}$. $\mathrm{O}$ agachamento afundo foi dividido em duas etapas, diferindo apenas o posicionamento do membro inferior dominante (randomizado). Os sinais eletromiográficos foram captados utilizando um eletromiógrafo e analisados os valores "root mean square" (RMS) na fase concêntrica. Os resultados indicaram um aumento significativo do RMS em função do tempo para o membro inferior posicionado frontalmente e posteriormente $(p<0,001)$. No membro posicionado frontalmente, o aumento do RMS correspondeu a 50\% para o VL, 54\% para o VM e $48 \%$ para o BF. O membro posicionado posteriormente apresentou um aumento de $75 \%$ para o VL, $113 \%$ para o VM, $62 \%$ para o BF e $48 \%$ para o ST. O RMS também foi significativamente maior no músculo $\mathrm{VM}$ em relação ao ST no membro inferior posicionado anteriormente $(p=0,03)$ e em relação ao ST e BF no membro inferior posicionado posteriormente $(p=0,02)$. Não ocorreu interação significativa entre o efeito do tempo e músculo na atividade eletromiográfica. O RMS normalizado não apresentou diferenças estatisticamente significativas no que se refere ao posicionamento do membro inferior dominante. A atividade muscular foi semelhante em ambos os posicionamentos do membro inferior, apresentando maior aumento de ativação dos músculos VL e VM em relação ao BF e ST.

Palavras-chave: Exercício; Exercício resistido; Fadiga; Root mean square.

Abstract - The position of lower limbs during lunge is different from squat without clear understanding on differences in muscle activation. The objective was to compare the electromyographic activity of the vastus lateralis (VL), vastus medialis (VM), biceps femoris (BF) and semitendinosus (ST) during the execution of the lunge to exhaustion of the lower limb, changing position of the legs frontally and posteriorly. Nine active women participated of this study with a mean (SD) age of 22 (3.4) years and body mass $60.3(4.1) \mathrm{kg}$. The lunge was divided in two phases, changing the forward leg (random order). The electromyographic signals were captured by an eletromyograph, and the root mean square (RMS) values were analyzed during the concentric phase. There was a significant increase of the RMS in time for the LL positioned frontally and posterior $(p<0,001)$. For the forward position, the increase of RMS was 50\% for VL, 54\% for VM and 48\% for BF. The backward position had an increase of $75 \%$ for VL, 113\% for VM, 62\% for BF and $48 \%$ for ST. The RMS was significantly higher for VM than for ST the forward position $(p=0,03)$ and ST and BF for the backward position $(p=0,02)$. No interaction was observed between time and muscle in the electromyigraphic activity. The normalized RMS was not statistically different comparing the forward and backward position of the dominant leg. Muscle activity was similar in both positions of the LL, showing increased activation of the VM and VL muscles in relation to the BF and ST. Key words: Exercise; Fatigue; Resistance exercise; Root mean square.
1 Universidade Federal de São Carlos. Laboratório de Fisiologia do Exercício. São Carlos, SP. Brasil.

2 Universidade de São Paulo. Programa de Pós-Graduação Interunidades Bioengenharia. São Carlos, SP. Brasil.

3 Universidade Federal de Ouro Preto. Centro Desportivo. Laboratório de Biomecânica e Cinesiologia. Ouro Preto, MG. Brasil.

Recebido em 24/09/10 Revisado em 29/09/11 Aprovado em 18/10/11

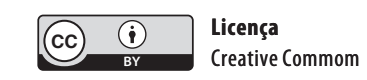




\section{INTRODUÇÃO}

A fadiga tem sido frequentemente descrita como um processo fisiológico tempo dependente que engloba numerosos fatores centrais e periféricos ${ }^{1}$, afetando a capacidade de produção de força e prejudicando o desempe$n^{n h o}{ }^{2,4}$. Todos estes mecanismos de fadiga são dependentes de cada tarefa ${ }^{5}$. Entretanto, os mecanismos periféricos de fadiga muscular resultam em diminuição da capacidade da célula muscular em gerar força, necessitando um aumento no estímulo neural para manter o trabalho mecânico constante ${ }^{6,8}$. Este aumento no estímulo neural é expresso no aumento do sinal eletromiográfico dos músculos em exercício ${ }^{6}$.

O agachamento é um exercício no qual o processo de fadiga está bem demonstrado, por meio da coativação do complexo muscular quadríceps e isquiotibiais para promover estabilização à articulação do joelho. $\mathrm{O}$ processo de fadiga diminui a força de contratilidade e o tempo de ativação muscular dos músculos do quadríceps, desencadeando o aumento da atividade muscular dos músculos isquiotibiais ${ }^{9,10}$. Tem sido muito frequente $\mathrm{o}$ emprego de variações desse exercício com o intuito de observar alterações nos padrões de atividade muscular, principalmente, em diferentes posicionamentos dos pés e ângulos de agachamento ${ }^{11-13}$.

Uma das variações mais utilizadas é o agachamento afundo, exercício de cadeia fechada amplamente prescrito devido à menor tensão exercida sobre o ligamento cruzado anterior ${ }^{2,14,15}$ comparado a exercícios de cadeia aberta. No entanto, questões sobre participações musculares e desenvolvimento de fadiga no agachamento afundo devem ser esclarecidas, uma vez que o movimento possui um posicionamento dos membros inferiores diferente do agachamento padrão. Pincivero et al..$^{16}$ demonstraram um aumento significativo de ativação muscular dos músculos vastus lateralis (VL), vastus medialis (VM) e bíceps femuralis $(\mathrm{BF})$ durante a realização do agachamento afundo até a fadiga. Entretanto, pouco se sabe sobre a eficácia do exercício em relação ao membro inferior posicionado posteriormente, uma vez que não foram encontrados estudos que tenham comparado a ativação muscular dos membros inferiores posicionados anteriormente e posteriormente no movimento de agachamento afundo.

O objetivo do estudo foi comparar a atividade eletromiográfica dos músculos VL, VM, BF e semitendinosus (ST) durante a execução do exercício agachamento afundo até a exaustão dos membros inferiores posicionados frontalmente e posteriormente. A análise eletromiográfica foi realizada apenas no membro dominante, alternando a sua posição de análise, ou seja, analisado nas posições anterior e posterior.

\section{PROCEDIMENTOS METODOLÓGICOS}

\section{Amostra}

Participaram do estudo nove sujeitos do sexo feminino, praticantes de atividade física, com média (DP) de idade $22(3,4)$ anos, estatura 163,4 $(4,6)$ 
$\mathrm{cm}$, massa corporal 60,3 $(4,1) \mathrm{kg}$ e $26,3(2,4) \%$ de percentual de gordura. O teste de PAR-Q (Physical Activity Readiness Questionnaire) foi aplicado para identificar os indivíduos saudáveis e excluir os que possuíssem alguma lesão que impossibilitassem a realização do movimento, assim como doença cardiopulmonar, sistêmica ou cerebral (American College of Sports Medicine, 1995). Todos os participantes leram e assinaram um consentimento livre e esclarecido aprovado pelo Comitê de Ética local. O projeto de pesquisa foi aprovado pelo Comitê de Ética da Universidade Federal de São Carlos, UFSCAR, 494-2009.

\section{Protocolo de exercício}

As coletas dos dados aconteceram em três etapas. A primeira etapa consistiu de uma avaliação da composição corporal por meio da balança de bioimpedância (Body Composition Analyser TBF-310, Tanita, Arlington Heights, IL, USA). Logo após, o membro inferior dominante foi determinado por meio de questionamento aos sujeitos de qual membro inferior este se utiliza preferencialmente para chutar uma bola. Os participantes realizaram uma breve execução do movimento para adaptação e determinação da distância entre os membros inferiores, que foi anotada para utilização nos testes seguintes. A distância do posicionamento dos membros inferiores foi determinada individualmente, correspondendo à medida entre a espinha ilíaca anterossuperior e o maléolo medial.

$\mathrm{Na}$ segunda e terceira etapa, foi aplicado o protocolo de teste do agachamento afundo, diferindo apenas o posicionamento do membro inferior dominante. $\mathrm{O}$ membro inferior dominante foi posicionado de forma randomizada nas duas sessões, permitindo a realização do protocolo de teste com o membro dominante posicionado anterior e posteriormente. As duas sessões foram necessárias para que a análise eletromiográfica fosse realizada apenas no membro inferior dominante, evitando as diferenças de ativação muscular entre membros. A segunda e terceira sessão foram realizadas no mesmo horário do dia com uma semana de intervalo.

Os sinais eletromiográficos de superfície foram captados nos músculos VL, VM, BF e ST do membro inferior dominante durante todo o protocolo de teste. Um ciclo do movimento foi definido como o período em que o sujeito se deslocava da posição estática com os joelhos em extensão até a posição de agachamento afundo (aproximadamente 90 graus de flexão do joelho anterior) e retorna a posição inicial. O participante realizou o movimento sem carga externa, com as mãos na cintura e em postura ereta (Figura 1). Para identificação do ângulo de flexão do joelho, foi utilizado um eletrogoniômetro acoplado ao eletromiógrafo, com visualização do ângulo articular em tempo real. Uma plataforma foi usada para apoio do joelho do membro inferior posicionado posteriormente, de forma a auxiliar o ângulo de agachamento do exercício, assim como minimizar riscos de lesão.

Todos os participantes foram incentivados a realizar o maior número de repetições, chegando à exaustão. Cada ciclo de movimento tinha a duração de 3 segundos (incluindo as fases concêntrica e excêntrica), realizando 20 
repetições por minuto. A velocidade de execução foi controlada com um cronômetro por um pesquisador, que transmitia sinais visuais e auditivos ao participante. A interrupção do teste seguiu alguns critérios: parada voluntária, falha concêntrica, falta de ar, tonturas ou outras situações que impossibilitassem a correta biomecânica do movimento.

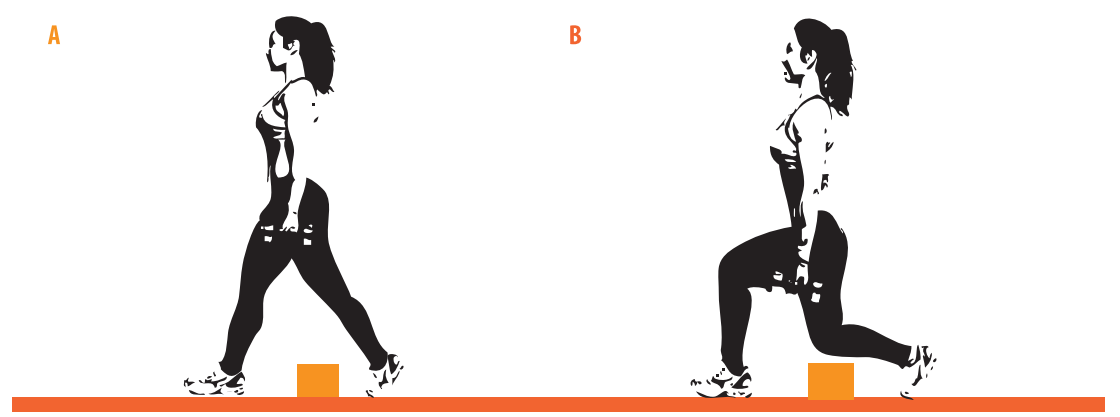

Figura 1. Representação esquemática da posição inicial (A) e final (B) do agachamento afundo.

A duração do exercício e o número de repetições total em cada sessão foram coletados. A frequência cardíaca (FC) foi continuamente mensurada utilizando um monitor cardíaco (Polar S610, Kempele, Filândia) e as medidas obtidas em repouso (um minuto antes do teste) e anterior à exaustão foram utilizadas para a análise dos dados.

\section{Aquisição dos sinais de eletromiografia e eletrogoniometria}

Os registros eletromiográficos foram coletados por um módulo de aquisição de sinais biológicos EMG System do Brasil (São José dos Campos, SP, Brasil) de oito canais, ao qual foram conectados os eletrodos. A frequência de amostragem foi de $1000 \mathrm{~Hz}$, sendo estabelecido um ganho de 1000 vezes. Também foi aplicado um filtro passa alta de $20 \mathrm{~Hz}$ e um filtro passa baixa de $500 \mathrm{~Hz}$.

Foram utilizados eletrodos de superfície (Ag-Ag/Cl - HAL;, São Paulo, SP, Brasil) com distância intereletrodo de $20 \mathrm{~mm}$. Antes da colocação dos eletrodos nos músculos, foi realizada a tricotomia com lâmina descartável, seguida de limpeza com álcool e algodão seco conforme recomendação da Sociedade Internacional de Eletromiografia e Cinesiologia ${ }^{17}$.

O posicionamento dos eletrodos também seguiu às normas da Sociedade Internacional de Eletromiografia e Cinesiologia ${ }^{17}$, correspondendo ao melhor ponto de captação para o músculo, sendo: (1) VL, 2/3 da linha entre a espinha ilíaca anterossuperior e a face lateral da patela, e os eletrodos colocados na direção da fibra muscular; (2) VM, 80\% da distância da linha medial desde a espinha ilíaca anterossuperior até o epicôndilo lateral do fêmur; e (3) ST e BF, 50\% da distância entre a tuberosidade isquiática para os epicôndilos medial e lateral do fêmur, respectivamente. O eletrodo de referência foi posicionado no processo estilóide da ulna.

Foi utilizado um eletrogoniômetro (EMG System do Brasil, São José dos Campos, SP, Brasil) para identificação da fase concêntrica e excêntrica do exercício, permitindo a análise do sinal eletromiográfico, separadamente, em cada fase do movimento. 


\section{Análises dos sinais de eletromiografia e eletrogoniometria}

A avaliação eletromiográfica foi realizada por meio da análise do root mean square (RMS) fase concêntrica do movimento dos músculos supracitados. A média das três primeiras fases concêntricas do movimento em cada protocolo foi utilizada para determinar o RMS inicial. O valor de RMS em cada fase concêntrica foi dividido pelo tempo de execução para obter uma média de um segundo (mV/s). Cada fase concêntrica compreendeu o movimento do participante desde a saída da posição do joelho flexionado a 90 graus até à completa extensão do joelho, controlado com o eletrogoniômetro. Finalmente, a média dos três valores originou um valor inicial por amostra para cada músculo. Esse valor foi considerado $100 \%$ da ativação muscular para os quatro músculos. Os valores de RMS foram então normalizados, dividindo cada valor pelo valor inicial obtido no início do exercício. Assim, todos os valores de RMS subsequentes durante o exercício na fase concêntrica foram convertidos para uma percentagem do valor de RMS obtido no início do exercício. Este método de normalização foi escolhido para comparar as diferenças percentuais em relação ao início do exercício para cada músculo estudado.

\section{Análise estatística}

O tamanho do efeito $\left(f^{2}\right)$ dos dados deste estudo foi igual a 0,70 , calculado para um poder da amostra (sample power; $1-\beta$ ) igual a 0,80 . Segundo as convenções de Cohen para o valor de $\mathrm{f}^{2}$, o tamanho do efeito é considerado grande. Os dados são apresentados em média (DP). Foi aplicado o teste-t de student para amostras pareadas para determinar as diferenças entre $o$ tempo de execução, número de repetições, FC e RMS normalizado de cada músculo entre as duas posições do membro inferior. O teste ANOVA de dupla entrada (tempo e músculo) foi utilizado para determinar as diferenças no valor de RMS em cada posição do membro inferior no agachamento afundo. O nível de significância aceito foi $p<0.05$ e o software estatístico utilizado foi o SPSS versão 17.0 (Somers, NY, USA).

\section{RESULTADOS}

Como observado na tabela 1 , não foram encontradas diferenças estatisticamente significativas em relação ao desempenho e resposta de frequência cardíaca das voluntárias, na execução dos protocolos de exercício com o membro inferior dominante, posicionado anteriormente e posteriormente.

Tabela 1. Média e desvio padrão do tempo, repetições e frequência cardíaca do agachamento afundo com o membro inferior posicionado frontalmente e posteriormente.

\begin{tabular}{lllll}
\hline & Tempo $(\mathrm{s})$ & Repetições & FC inicial (bpm) & \multicolumn{1}{l}{$\begin{array}{l}\text { FC final } \\
(\mathrm{bpm})\end{array}$} \\
\hline MIPF & $247,2(73,6)$ & $82,8(24,5)$ & $107,0(5,5)$ & $165,5(9,5)$ \\
MIPP & $226,0(73,2)$ & $69,2(20,4)$ & $107,8(4,2)$ & $165,3(11)$ \\
\hline
\end{tabular}

Legenda: $\mathrm{MIPF}=$ membro inferior dominante posicionado frontalmente; $\mathrm{MIPP}=$ membro inferior dominante posicionado posteriormente; $\mathrm{FC}$ = frequência cardíaca. 
Os valores RMS normalizados de cada músculo durante a realização do agachamento afundo com o membro inferior dominante posicionado frontalmente são apresentados na figura 2. Não ocorreu interação significativa entre o efeito do tempo e os valores de RMS normalizados dos diferentes músculos, $F(15,192)=0,57, p=0,90$. A ausência de interação determina que os músculos estudados apresentaram um padrão de ativação muscular semelhante durante o exercício. Os músculos VL, VM e $\mathrm{BF}$ apresentaram um aumento significativo na ativação muscular com o tempo de exercício, $F(5,192)=8,18, p<0,001$. O aumento correspondeu a, aproximadamente, $50 \%$ para o músculo VL, $54 \%$ para o VM e $48 \%$ para o BF. O RMS normalizado foi significativamente maior no músculo VM em relação ao ST, após a realização de $20 \%$ do exercício, $F(3,15)=3,09, p=0,03$.

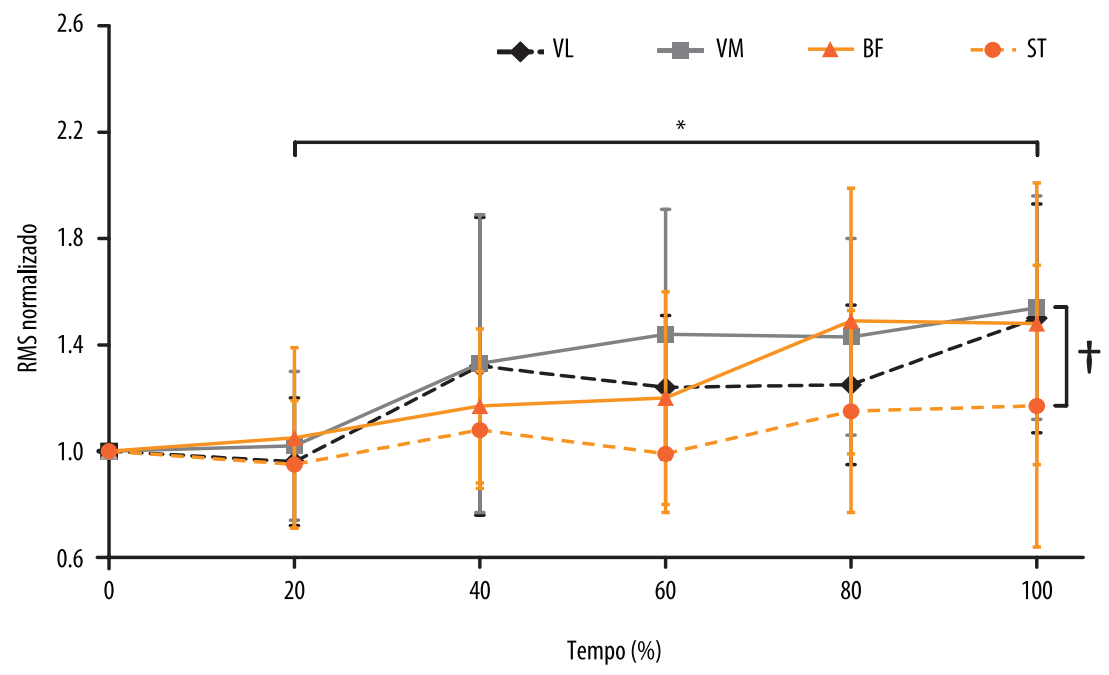

Figura 2. RMS normalizado da atividade eletromiográfica dos músculos VL (vastus lateralis), VM (vastus medialis), BF (bíceps femuralis) e ST (semitendinosus) durante 0 exercício de agachamento afundo com MI posicionado frontalmente. *Diferença significativa para os músculos VM, VL e BF entre o momento $20 \mathrm{e}$ $100 \%$. †Diferença significativa entre os músculos VM e ST nos momentos 20, 40, 60, 80 e 100\% ( $p<0,05)$.

A figura 3 apresenta os valores normalizados do RMS para cada músculo durante a realização do agachamento afundo com o membro inferior dominante posicionado posteriormente. Também não ocorreu interação significativa entre o efeito tempo e os diferentes músculos nos valores de RMS normalizados, $F(15,184)=1,02, p=0,44$, sugerindo um padrão de aumento semelhante entre os músculos. A ativação muscular apresentou um aumento significativo com o tempo de exercício em todos os músculos analisados, $F(5,184)=15,61, p<0,001$. Os músculos VL, VM, BF e ST apresentaram um aumento de 75\%, 113\%, 62\% e 43\% em relação ao início do exercício, respectivamente. O RMS normalizado também foi significativamente maior no músculo $\mathrm{VM}$ em relação ao $\mathrm{BF}$ e ST em $80 \%$ e no final do exercício, $F(3,15)=3,56, p=0,02$.

Não foram observadas diferenças significativas entre os valores de RMS normalizados relativamente ao posicionamento do membro inferior. Independentemente do posicionamento do membro inferior dominante neste exercício, o valor de RMS normalizado do VL, VM, BF e ST foi semelhante nas duas posições. 


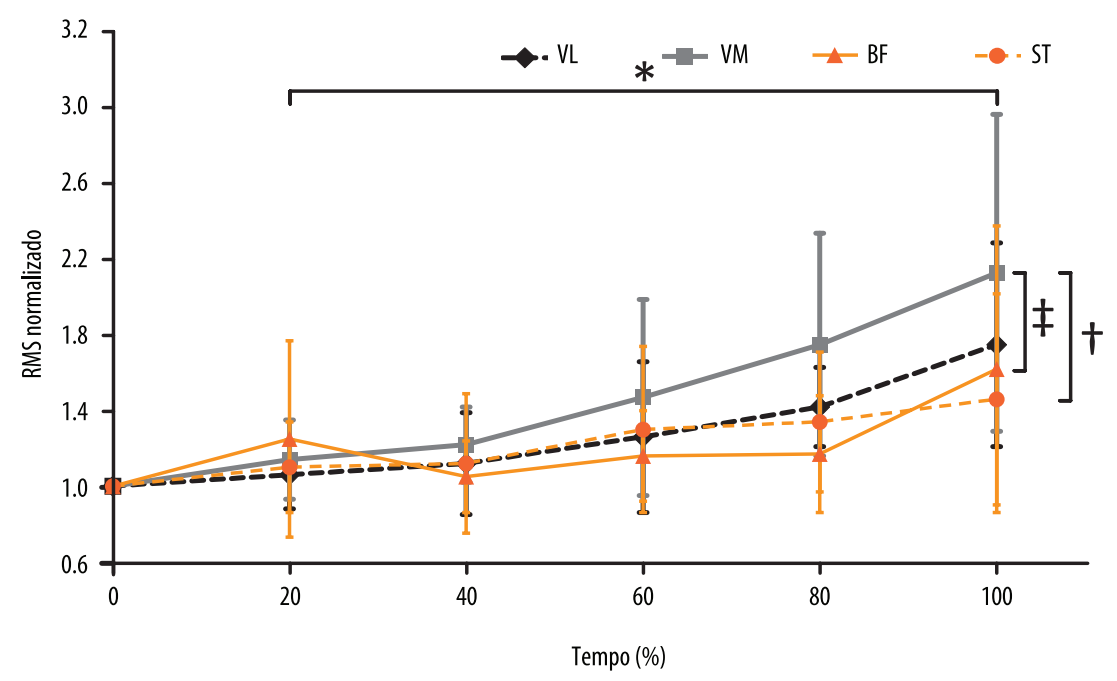

Figura 3. RMS normalizado da atividade eletromiográfica dos músculos VL (vastus lateralis), VM (vastus medialis), BF (bíceps femuralis) e ST (semitendinosus)durante o exercício agachamento afundo com Ml posicionado posteriormente. *Diferença significativa para todos os músculos entre o momento 20 e 100\%. †Diferença significativa entre os músculos VM e ST no momento 80 e 100\%. ‡Diferença significativa entre os músculos VM e BF no momento 80 e 100\% $(p<0,05)$.

\section{DISCUSSÃO}

Os resultados deste estudo demonstram maior ativação dos músculos anteriores da coxa em relação aos posteriores, durante a realização do agachamento afundo em função do tempo. Os músculos extensores do joelho são recrutados preferencialmente durante o agachamento e, por essa razão, apresentam maiores indícios de fadiga muscular, observado pelo aumento percentual do RMS. Durante a avaliação do agachamento afundo no membro inferior dominante posicionado frontalmente, os músculos VL, VM e BF apresentam um percentual de aumento de ativação mais evidente que o músculo ST. Especificamente, não ocorreu alteração da ativação do músculo ST no membro inferior dominante posicionado frontalmente na realização do agachamento afundo. No entanto, quando o membro inferior dominante posicionado posteriormente foi avaliado, todos os músculos apresentaram um aumento significativo. $\mathrm{O}$ estudo também demonstra a ausência de diferença de ativação entre o membro posicionado anteriormente e posteriormente, mostrando uma ativação muscular semelhante entre as duas posições.

No membro inferior posicionado frontalmente, os músculos VL e VM apresentaram um comportamento similar durante a fase concêntrica do movimento, como demonstrado por meio da análise visual da figura 2. Estes resultados sugerem que os músculos VL e VM agem como sinergistas durante este exercício, como descrito por Pincivero et al. ${ }^{16} \mathrm{O}$ músculo $\mathrm{BF}$ também apresentou um aumento na sua atividade, no entanto, a sua ativação é entendida como coativação ${ }^{16}$, apresentando função principal quando os músculos do quadríceps começam a entrar em processo de fadiga. No presente estudo, a ativação do músculo BF começou a aumentar em, aproximadamente, $60 \%$ do tempo total de exercício, resultado semelhante descrito 
por Pincivero et al. ${ }^{16}$ Mudanças na ativação do BF podem ser explicadas por um mecanismo de proteção dinâmica, a fim de minimizar o impacto nas articulações, principalmente do joelho ${ }^{2,14,18}$. A ativação do BF pode ser entendida como coativação muscular, representando um fator importante para a estabilidade dinâmica ${ }^{19,20}$, ocorrendo concomitantemente com o processo de fadiga ${ }^{16,21}$. Por outro lado, o valor de RMS do músculo ST não aumentou significativamente durante o agachamento afundo. Van Lent et al. ${ }^{22}$ demonstraram que, durante uma caminhada, o músculo ST é ativado excentricamente apenas na fase final da extensão do membro inferior de forma a minimizar a rotação externa do joelho. Como a análise eletromiográfica foi realizada apenas na fase concêntrica, sua ativação pode ter ficado fora da janela do tempo onde os dados foram processados e analisados.

Surpreendentemente, os resultados do membro inferior posicionado posteriormente são muito semelhantes ao membro inferior posicionado frontalmente. Os músculos sinergistas do movimento continuam a ser o VM e VL, ocorrendo, também, coativação dos músculos BF e do ST. De acordo com o estudo de Jonhagen et al., ${ }^{23}$ o quadríceps é o músculo ativo durante as primeiras fases do agachamento e, para manter a postura do movimento, os ísquiotibiais apresentam uma contração isométrica e o gastrocnêmio são ativados excentricamente. Tal conclusão, no entanto, não pode ser estendida para o presente estudo, pois o gastrocnêmio não foi avaliado.

O músculo VM foi o que apresentou maior diferença de ativação entre as duas posições estudadas, aumentando mais de 100\% quando posicionado posteriormente e apenas $50 \%$ na posição frontal. Entretanto, embora o sinal eletromiográfico normalizado seja semelhante entre as posições, é importante destacar que essa ativação pode não representar o mesmo nível de esforço, mesmo após a sua normalização ${ }^{24}$. Vários estudos já demonstraram que a relação entre o sinal eletromiográfico e força não é linear. ${ }^{25,26} \mathrm{~A}$ não linearidade pode ser devido a uma diferença entre as propriedades elétricas ou tensões específicas de diferentes tipos de unidades motoras $^{25,26}$. Deste modo,pode-se afirmar apenas que ocorreu um aumento da ativação muscular durante o exercício nas duas posições, inclusive, para os músculos anteriores da coxa quando o membro inferior está posicionado posteriormente. Mesmo nesta posição, pode ocorrer o processo de fadiga no VL e VM, afetando diretamente o desempenho se o agachamento for realizado nas duas posições sem um adequado intervalo de recuperação. Estes achados demonstram a importância da individualização e direcionamento do agachamento afundo em um treinamento. A pré-exaustão dos músculos anteriores de coxa pode condicionar diretamente o desempenho do exercício.

Outras técnicas de análise eletromiográfica, conjugadas com a análise do RMS, poderiam ter elucidado a relação entre fadiga muscular e força desenvolvida nos diferentes músculos. Apesar do aumento do sinal RMS, o que poderá ser indicativo do processo de fadiga durante a tarefa proposta, a análise do RMS mostrou-se insuficiente para discutir as diferenças entre a ativação e força muscular nas diferentes posições. Recomendam-se novas 
pesquisas para estabelecer a relação entre a ativação muscular e os dois posicionamentos do membro inferior.

Apesar da limitação da análise, a realização de uma série do agachamento afundo com um determinado membro inferior posicionado frontalmente, seguida de nova série de exercício com troca de membro inferior, poderá ser entendida como uma pré-exaustão nos músculos do membro inferior que iniciou o exercício posteriormente. Dessa forma, é promovido um maior nível de fadiga muscular ao membro inferior que iniciou o movimento posicionado posteriormente, favorecendo um estímulo diferenciado. A avaliação da pré-exaustão em estudos futuros faz-se necessária para determinar os efeitos na fadiga muscular durante este exercício específico.

\section{CONCLUSÃO}

No exercício de agachamento afundo parece ocorrer maior ativação muscular do VL e VM em relação ao BF e ST. Quando esse tipo de exercício é realizado até a exaustão, o aumento da atividade eletromiográfica dos músculos agonistas parece ser semelhante, independentemente do posicionamento do membro inferior. Esses achados sugerem que ocorra uma pré-exaustão nos grupos musculares do membro inferior que inicia a série de exercícios posicionado posteriormente, o que pode limitar seu desempenho durante a posterior realização do agachamento afundo posicionado anteriormente.

\section{REFERÊNCIAS BIBLIOGRÁFICAS}

1. Sahlin K. Metabolic factors in fatigue. Sports Med 1992;13(2):99-107.

2. Palmitier RA, An KN, Scott SG, Chao EY. Kinetic chain exercise in knee rehabilitation. Sports Med 1991;11(6):402-13.

3. Lutz GE, Palmitier RA, An KN, Chao EY. Comparison of tibiofemoral joint forces during open-kinetic-chain and closed-kinetic-chain exercises. J Bone Joint Surg Am 1993;75(5):732-9.

4. Stuart MJ, Meglan DA, Lutz GE, Growney ES, An KN. Comparison of intersegmental tibiofemoral joint forces and muscle activity during various closed kinetic chain exercises. Am J Sports Med 1996;24(6):792-9.

5. Enoka RM, Stuart DG. Neurobiology of muscle fatigue. J Appl Physiol 1992;72(5):1631-48.

6. Gerdle B, Karlsson S, Crenshaw AG, Friden J. The relationships between EMG and muscle morphology throughout sustained static knee extension at two submaximal force levels. Acta Physiol Scand 1997;160(4):341-51.

7. Mannion AF, Dolan P. Relationship between myoelectric and mechanical manifestations of fatigue in the quadriceps femoris muscle group. Eur J Appl Physiol Occup Physiol 1996;74(5):411-9.

8. Vaz MA, Zhang YT, Herzog W, Guimaraes AC, MacIntosh BR. The behavior of rectus femoris and vastus lateralis during fatigue and recovery: an electromyographic and vibromyographic study. Electromyogr Clin Neurophysiol 1996;36(4):221-30.

9. Andriacchi TP, Birac D. Functional testing in the anterior cruciate ligamentdeficient knee. Clin Orthop Relat Res 1993(288):40-7.

10. Hurwitz DE, Andriacchi TP, Bush-Joseph CA, Bach BR, Jr. Functional adaptations in patients with ACL-deficient knees. Exerc Sport Sci Rev 1997;25:1-20. 
11. Escamilla RF, Fleisig GS, Zheng N, Lander JE, Barrentine SW, Andrews JR, et al. Effects of technique variations on knee biomechanics during the squat and leg press. Med Sci Sports Exerc 2001;33(9):1552-66.

12. Lam PL, Ng GY. Activation of the quadriceps muscle during semisquatting with different hip and knee positions in patients with anterior knee pain. Am J Phys Med Rehabil. 2001;80(11):804-8.

13. Hertel J, Sloss BR, Earl JE. Effect of foot orthotics on quadriceps and gluteus medius electromyographic activity during selected exercises. Arch Phys Med Rehabil 2005;86(1):26-30.

14. Draganich LF, Jaeger RJ, Kralj AR. Coactivation of the hamstrings and quadriceps during extension of the knee. J Bone Joint Surg Am 1989;71(7):1075-81.

15. Henning CE, Lynch MA, Glick KR, Jr. An in vivo strain gage study of elongation of the anterior cruciate ligament. Am J Sports Med 1985;13(1):22-6.

16. Pincivero DM, Aldworth C, Dickerson T, Petry C, Shultz T. Quadriceps-hamstring EMG activity during functional, closed kinetic chain exercise to fatigue. Eur J Appl Physiol 2000;81(6):504-9.

17. Hermens HJ, Freriks B, Disselhorst-Klug C, Rau G. Development of recommendations for SEMG sensors and sensor placement procedures. J Electromyogr Kinesiol 2000;10(5):361-74.

18. Solomonow M, Baratta R, Zhou BH, Shoji H, Bose W, Beck C, et al. The synergistic action of the anterior cruciate ligament and thigh muscles in maintaining joint stability. Am J Sports Med 1987;15(3):207-13.

19. Shields RK, Madhavan S, Gregg E, Leitch J, Petersen B, Salata S, et al. Neuromuscular control of the knee during a resisted single-limb squat exercise. Am J Sports Med 2005;33(10):1520-6.

20. Souza CO, Ferreira JJA, Medeiros ACLV, Carvalho AH, Pereira RC, Guedes DT, et al. Atividade eletromiográfica no agachamento nas posições de $40^{\circ}, 60^{\circ}$ e $90^{\circ}$ de flexão do joelho. Rev Bras Med Esporte 2007;13(5):306-16.

21. Marson RA, Gonçalves M. Electromyographic behavior of the biceps femoris (caput longum) and Semitendinosus muscles in the isometric contraction test. Brazilian Journal of Morphological Sciences 2003;20(1):55-8.

22. van Lent ME, Drost MR, vd Wildenberg FA. EMG profiles of ACL-deficient patients during walking: the influence of mild fatigue. Int J Sports Med 1994;15(8):508-14.

23. Jonhagen S, Halvorsen K, Benoit DL. Muscle activation and length changes during two lunge exercises: implications for rehabilitation. Scand J Med Sci Sports 2009;19(4):561-8.

24. De Luca CJ. The use of surface electromyography in biomechanics. J Appl Biomech 1997;13(2):135-63.

25. Siegler S, Hillstrom HJ, Freedman W, Moskowitz G. The effect of myoelectric signal processing on the relationship between muscle force and processed EMG. Electromyogr Clin Neurophysiol 1985;25(7-8):499-512.

26. Bigland-Ritchie B. EMG/force relations and fatigue of human voluntary contractions. Exerc Sport Sci Rev 1981;9:75-117.
Endereço para correspondência

Gisele Rodrigues Leite Garcia

Rio Grande do Sul, 256

CEP 37701-001 - Poços de Caldas,

MG. Brasil

E-mail: gi.garcia@hotmail.com 